\title{
Improving queuing system with limited resources using TRIZ and arena simulation
}

\begin{abstract}
A university cafeteria is a queueing system characterised by non-stationary time of arrival with limited resources where the arrival rate is time dependent and has different pattern of arrival for different time interval. This means at certain time of the day; the arrival rate is much higher than other time. For a university cafeteria, the arrival rate of customer during the lunchtime is higher and the food (resources) is limited. Non-stationary time dependent queueing systems are not easily modelled mathematically hence such queueing systems are modelled using simulation tools such as ARENA. In order to model a non-stationary time dependent queueing system with limited resources and solve queueing problems using ARENA, researchers have to rely on their knowledge and experience to identify the appropriate parameters of the system and make modifications to these parameters of the system to solve queueing problems by means of trial and error. Hence, this research work explores the potentials of applying a systematic problem solving tool, TRIZ to help users to make better decisions in deriving solutions to improve a non-stationary time dependent queueing system with limited resources. A case study was carried out to minimize the waiting time of the customers at the cafeteria of the Faculty of Engineering, Universiti Putra Malaysia (UPM), which has queueing problems for years during lunchtime. TRIZ was applied in this case study and the results showed that TRIZ can assist researchers to derive a solution model that leads to shorter waiting time without incurring additional cost and resources.
\end{abstract}

Keyword: Arena software; Average waiting time; Queuing problem; TRIZ 